ARTÍCULO DE INVESTIGACIÓN

\title{
Comparación del contenido de compuestos fenólicos en la corteza de ocho especies de pino
}

\author{
Martha Rosales Castro ${ }^{1}$ \\ Rubén F. González Laredo
}

\begin{abstract}
RESUMEN
Se evaluó el contenido de taninos condensados y fenoles totales, expresados como ácido tánico, en extractos etanólicos y acuosos de las cortezas de ocho especies de pino abundantes en el estado de Durango. Los extractos etanólicos se obtuvieron por maceración durante $48 \mathrm{~h}$ con etanol acuoso al $50 \%$ y los acuosos con agua, a ebullición y reflujo. La evaluación de taninos condensados se realizó mediante el número de Stiasny y los fenoles totales se evaluaron por el método de Folín-Ciocalteu utilizando ácido tánico como estándar. Los rendimientos en extracto o sólidos totales extraídos, Stiasny, taninos condensados y fenoles fueron mayores en los extractos etanólicos que en los acuosos, en todas las especies. Se encontraron diferencias estadísticas en la concentración de compuestos fenólicos entre los solventes de extracción utilizados y entre las especies. Los extractos etanólicos de las cortezas de Pinus leiophylla, $P$. ayacahuite, $P$. durangensis y $P$. teocote presentaron la mayor concentración de taninos condensados y fenoles, mientras que las especies $P$. cooperi y $P$. engelmannii presentaron la menor concentración.
\end{abstract}

PALABRAS CLAVE:

Corteza de pino, fenoles totales, Folín-Ciocalteu, taninos condensados, Stiasny.

\begin{abstract}
Condensed tannins and total phenols content expressed as tannic acid were evaluated on ethanolic and aqueous extracts from bark of eight Pinus species abundant at Durango State forests. Ethanolic extracts were obtained by soaking bark $48 \mathrm{~h}$ with 50 $\%$ aqueous ethanol and the aqueous extracts with hot water. Condensed tannins were evaluated by the Stiasny number and total phenols by the Folín-Ciocalteu method. Extract yields, Stiasny number, condensed tannins and total phenols were higher in ethanolic extracts, for all species. Statistical differences were found between extraction solvents and species. Ethanolic extracts of $P$. leiophylla, $P$. ayacahuite, $P$. durangensis and $P$. teocote barks showed the highest concentration of condensed tannins and phenols, while $P$. cooperi and $P$. engelmannii showed the lowest.
\end{abstract}

KEY WORDS:

Pinus bark, total phenols, Folín-Ciocalteu, condensed tannins, Stiasny. 


\section{INTRODUCCIÓN}

El estado de Durango es uno de los principales productores de madera en México, donde las especies más explotadas son los pinos. Existen aproximadamente veinte especies de pino en el estado, de las cuales diez son las más aprovechadas, principalmente por su abundancia y calidad de madera, entre las que se encuentran Pinus durangensis, Pinus arizonica, Pinus engelmannii y Pinus cooperi (García y González, 1998). Otras especies representativas son Pinus leiophylla, Pinus ayacahuite y Pinus chihuahuana. Del aprovechamiento de la madera se generan altos volúmenes de corteza, ya que ésta representa del $10 \%$ al $15 \%$ del peso total del árbol (Kofujita et al., 1999) y aunque es un recurso abundante se subutiliza, empleándolo como combustible.

La corteza, al igual que la madera, está formada químicamente por los componentes típicos de la pared celular: celulosa, lignina y por sustancias extraíbles, que se forman a partir del metabolismo secundario de las plantas y que contienen compuestos variados como terpenos, grasas, ceras, fenoles y azúcares, entre otros (Fengel y Wegener, 1989). Esta composición química depende de factores diversos como edad, especie, condiciones del árbol y localización (Vázquez et al., 1987). Un grupo importante de estos compuestos por su abundancia, diversidad y complejidad son los fenoles. Existe una gran variedad de estos compuestos, entre ellos: fenoles simples, ácidos fenólicos, cumarinas, estilbenos, flavonoides, lignanos y taninos (Dey y Harborne, 1989).

El uso tradicional de los fenoles vegetales ha sido en la formulación de adhesivos y en el curtido de pieles (Garro et al., 1997; Van Sumere, 1989); sin embargo, su utilización puede ser muy amplia, como en las áreas biomédicas, en las que se aprovechan las propiedades antimicrobianas, antioxidantes, antiinflamatorias y anticancerígenas (De Bruyne et al., 1999; Peterson y Dwyer, 2000), que les otorgan, principalmente, los flavonoides.

La importancia de evaluar la concentración de compuestos fenólicos entre diversas especies forestales radica en conocer cuales de ellas pueden ser las fuentes más importantes de estos compuestos. Los compuestos fenólicos pueden extraerse de material vegetal utilizando diversos solventes: acetona, etanol, metanol, agua, así como mezclas de ellos en diferentes proporciones (Waterman y Mole, 1994), siendo el agua caliente el solvente más económico para la extracción (González et al., 1989).

\section{OBJETIVO}

Evaluar la concentración de compuestos fenólicos, expresados como taninos condensados y fenoles totales, en extractos etanólicos y acuosos de la corteza de ocho especies de pino, las posibles diferencias entre ellas y definir las especies en las que se tiene la mayor concentración de estos compuestos, para su posible utilización en áreas biomédicas.

\section{METODOLOGÍA}

Se seleccionó corteza de ocho especies de pino, Pinus arizonica, $P$. ayacahuite, $P$. cooperi, $P$. chihuahuana, $P$. durangensis, $P$. engelmannii, $P$. leiophylla y $P$. teocote. Las muestras fueron colectadas en el Municipio de Pueblo Nuevo Durango, excepto $P$. chihuahuana que se colectó en el km 36 de la carretera Durango-La Flor. Las especies fueron identificadas por personal del Herbario CIIDIR IPN Unidad Durango, en el que se depositaron los ejemplares botánicos. Se recolectó la corteza de cinco a seis 
árboles por especie, a diferentes alturas del árbol, se mezclaron y tomaron muestras representativas de cada una.

\section{Obtención de extractos}

Considerando que la utilización principal de los extractos obtenidos de las cortezas de este estudio se propone para áreas biomédicas (Rosales, 2002) y de acuerdo con lo que presentan Waterman y Mole (1994) y González et al. (1989), con respecto a los solventes y condiciones de extracción para compuestos fenólicos, los extractos se obtuvieron a partir de corteza seca y triturada, tamizada a un tamaño de partícula de 10 mallas. Se obtuvieron extractos en etanol acuoso al $50 \%$ y en agua caliente. En los extractos etanólicos se utilizaron $10 \mathrm{~g}$ de corteza y $100 \mathrm{ml}$ de etanol al $50 \%$, se maceraron a temperatura ambiente durante $24 \mathrm{~h}$ y se filtraron sobre papel filtro. A la corteza remanente se le adicionaron $100 \mathrm{ml}$ de solvente fresco y se repitió el proceso. Los extractos obtenidos de la primera y segunda maceración se combinaron y concentraron en rotavapor a $35{ }^{\circ} \mathrm{C}$ aplicando vacío, obteniendo un extracto acuoso concentrado, a partir del cual se realizaron los análisis. Para los extractos acuosos se utilizaron $20 \mathrm{~g}$ de corteza y $200 \mathrm{ml}$ de agua, se calentó a ebullición y reflujo durante $1 \mathrm{~h}$, se filtró sobre papel filtro. En ambos extractos se calculó el rendimiento en sólidos (extracto total), evaluado como el peso total de los sólidos extraídos entre la cantidad de muestra seca utilizada en cada extracción.

\section{Determinación de taninos}

Para la cuantificación de taninos se utilizó el método de Stiasny, como una forma indirecta de medir la concentración de compuestos flavonoides presentes, ya que de acuerdo con lo indicado por Yazaki et al. (1993); Yazaki y Hillis (1980), el Número de Stiasny corresponde a la cantidad de poliflavonoides o taninos condensados que reaccionan con formaldehído en medio ácido y, por definición, "los poliflavonoides o taninos condensados son polímeros multihidroxilados, basados en unidades flavonoides de 15 carbonos" (Pizzy y Mittal, 1994). El Número de Stiasny es un método gravimétrico que consiste en agregar $10 \mathrm{ml}$ de formaldehído al $38 \%$ y $5 \mathrm{ml}$ de ácido clorhídrico concentrado a $50 \mathrm{ml}$ de extracto. La mezcla se deja en ebullición y reflujo durante $30 \mathrm{~min}$, el precipitado formado se separa sobre un filtro de vidrio de porosidad media, se lava con agua caliente, se seca a peso constante y se cuantifica. En otros $50 \mathrm{ml}$ de extracto se determina la cantidad de sólidos totales, evaporándolos a sequedad y pesando. El número de Stiasny es la relación entre el precipitado formado con respecto a los sólidos totales y corresponde al porcentaje de taninos condensados en el extracto. El porcentaje de taninos condensados en la corteza se calcula multiplicando este número en fracción, por el rendimiento en sólidos obtenidos en cada extracto, de acuerdo con la siguiente fórmula:

$$
\text { No. Stiasny }=\frac{P P \cdot 100}{P R}
$$

donde:

$\mathrm{PP}=$ peso del precipitado, $\mathrm{g}$

$\mathrm{PR}=$ peso del residuo de $50 \mathrm{ml}$ de extracto, $\mathrm{g}$

$$
T C=\frac{N S \cdot E T}{100}
$$

donde:

$\mathrm{TC}=$ porcentaje de taninos condensados

NS = número de Stiasny

$\mathrm{ET}=$ porcentaje de extracto total 


\section{Determinación de fenoles (expresados como ácido tánico)}

El análisis de fenoles totales se realizó por el método de Folín-Ciocalteu, de acuerdo con lo establecido por Waterman y Mole (1994) y Scalbert (1992), utilizando ácido tánico como estándar, por lo que los resultados se expresan como porcentaje de ácido tánico en cada extracto.

Este es un método espectrofotométrico que consiste en agregar $0,1 \mathrm{ml}$ de muestra a un matraz de $10 \mathrm{ml}$ que contenga de $6 \mathrm{ml}$ a $7 \mathrm{ml}$ de agua desionizada, luego $0,5 \mathrm{ml}$ del reactivo comercial de Folín-Ciocalteu. Después de 1 min y antes de 8 min se agregan $1,5 \mathrm{ml}$ de $\mathrm{Na}_{2} \mathrm{CO}_{3}$ al $20 \%$, se afora a $10 \mathrm{ml}$ con agua desionizada, se mezcla y después de $2 \mathrm{~h}$ se mide la absorbancia a $760 \mathrm{~nm}$. Se preparó una curva estándar de 20 ppm a 200 ppm de ácido tánico.

\section{Diseño experimental}

Se trabajó con un diseño estadístico de bloques al azar, con ocho especies, dos extractos (tratamientos o sistemas de extracción) y tres repeticiones. Las diferencias se evaluaron mediante un análisis de varianza.

\section{RESULTADOS Y DISCUSIÓN}

Los resultados de los rendimientos promedio del porcentaje de extracto total, Número de Stiasny, taninos condensados y fenoles totales expresados como porcentaje de ácido tánico, en los extractos etanólicos y acuosos se presentan en la Tabla 1.
Los rendimientos en extracto total (sólidos totales extraídos) variaron con respecto al solvente de extracción y a la especie. Los rendimientos más bajos fueron los del extracto acuoso, obteniéndose valores de $2,23 \%(P$. cooperi) a $10,61 \%$ ( $P$. ayacahuite), mientras que en el extracto etanólico los rendimientos alcanzados fueron de $6,6 \%$ \% $(P$. engelmannii) a 19,4\% (P. ayacahuite). En todas las especies el rendimiento en extracto obtenido con etanol al $50 \%$ fue el doble o mayor al obtenido con agua. De acuerdo con el análisis estadístico existen diferencias significativas $(p<0,05)$ en el rendimiento en extracto total entre las especies y entre los solventes de extracción (tratamientos). En la Tabla 2 se presentan los valores medios del porcentaje de extracto total y sus diferencias estadísticas.

El rendimiento de extracto en $P$. durangensis es semejante al encontrado para esta especie al utilizarse como solvente etanol acuoso al $80 \%$ (Rosales et al., 2002), en el cual el rendimiento fue del $16,15 \%$, menor a $18,7 \%$, que es el rendimiento obtenido con el sistema de extracción acetona acuosa al $70 \%$.

Para algunas especies de pino se han encontrado rendimientos en extractos acuosos de $13,3 \%$ en $P$. radiata (Inoue et al., 1998); 4,1\% en P. contorta; $11,6 \%$ en extractos etanólicos de $P$. echinata (Vázquez et al., 1987) y $8,8 \%$ en extractos de acetona al $50 \%$ en $P$. loblolly (Laks y McKaig, 1988).

El número de Stiasny obtenido en los extractos etanólicos fue superior al de los extractos acuosos. El menor valor de Stiasny fue de $30 \%$ para el extracto acuoso de $P$. engelmannii, mientras que el mayor fue para el extracto etanólico de $P$. leiophylla con $85 \%$. 
Tabla 1. Rendimientos promedio de los diferentes extractos y especies

\begin{tabular}{|c|c|c|c|c|}
\hline $\begin{array}{l}\text { ESPECIE } \\
\text { EXTRACTO }\end{array}$ & $\begin{array}{c}\text { RENDIMIENTO } \\
\text { EXTRACTO } \\
\text { TOTAL, \% }\end{array}$ & $\begin{array}{l}\text { NO. DE } \\
\text { STIASNY, \% }\end{array}$ & $\begin{array}{c}\text { TANINOS } \\
\text { CONDENSADOS, \% }\end{array}$ & $\begin{array}{c}\text { FENOLES } \\
\text { COMO ÁCIDO } \\
\text { TÁNICO, \% }\end{array}$ \\
\hline \multicolumn{5}{|l|}{ Pinus arizonica } \\
\hline Etanólico & 9,66 & 67,0 & 6,46 & 61,2 \\
\hline Acuoso & 5,87 & 53,6 & 3,15 & 53,5 \\
\hline \multicolumn{5}{|c|}{ Pinus ayachuite } \\
\hline Etanólico & 19,39 & 80,0 & 15,50 & 61,83 \\
\hline Acuoso & 10,61 & 69,0 & 7,32 & 64,33 \\
\hline \multicolumn{5}{|l|}{ Pinus cooperi } \\
\hline Etanólico & 9,94 & 71,0 & 7,06 & 49,16 \\
\hline Acuoso & 2,23 & 44,4 & 1,00 & 37,5 \\
\hline \multicolumn{5}{|c|}{ Pinus chihuahuana } \\
\hline Etanólico & 8,86 & 81,2 & 7,20 & 81,7 \\
\hline Acuoso & 4,14 & 59,4 & 2,46 & 62,0 \\
\hline \multicolumn{5}{|c|}{ Pinus durangensis } \\
\hline Etanólico & 16,68 & 80,8 & 13,48 & 78,2 \\
\hline Acuoso & 9,32 & 69,3 & 6,46 & 72,2 \\
\hline \multicolumn{5}{|c|}{ Pinus engelmannii } \\
\hline Etanólico & 6,66 & 49,0 & 3,25 & 59,5 \\
\hline Acuoso & 3,30 & 30,0 & 1,00 & 32,2 \\
\hline \multicolumn{5}{|c|}{ Pinus leiophylla } \\
\hline Etanólico & 19,11 & 85,0 & 16,18 & 78,5 \\
\hline Acuoso & 7,35 & 80,0 & 5,88 & 72,2 \\
\hline \multicolumn{5}{|l|}{ Pinus teocote } \\
\hline Etanólico & 12,15 & 75,0 & 9,12 & 62,3 \\
\hline Acuoso & 5,27 & 57,4 & 3,02 & 56,8 \\
\hline
\end{tabular}


Tabla 2. Tabla de medias del porcentaje de extracto total

\begin{tabular}{llll}
\hline \multicolumn{2}{c}{ EXTRACTO ACUOSO } & \multicolumn{2}{c}{ EXTRACTO ETANÓLICO } \\
\hline Especie & Media ${ }^{*}$ & Especie & Media ${ }^{*}$ \\
\hline P. ayacahuite & $10,61 \mathrm{a}$ & P. ayacahuite & $19,39 \mathrm{a}$ \\
\hline P. durangensis & $9,32 \mathrm{~b}$ & P. leiophylla & $19,11 \mathrm{a}$ \\
\hline P. leiophylla & $7,35 \mathrm{c}$ & P. durangensis & $16,68 \mathrm{~b}$ \\
\hline P. arizonica & $5,87 \mathrm{~d}$ & P. teocote & $12,15 \mathrm{c}$ \\
\hline p. teocote & $5,27 \mathrm{e}$ & P. cooperi & $9,94 \mathrm{~d}$ \\
\hline P. chihuahuana & $4,14 \mathrm{f}$ & P. arizonica & $9,66 \mathrm{~d}$ \\
\hline P. engelmannii & $3,30 \mathrm{~g}$ & P. chihuahuana & $8,86 \mathrm{e}$ \\
\hline P. cooperi & $2,23 \mathrm{~h}$ & P. engelmannii & $6,65 \mathrm{f}$ \\
\hline
\end{tabular}

*Letras iguales significa que no existe diferencia estadística

Para extractos acuosos de la corteza de $P$. radiata se han encontrado valores de Stiasny de $81,5 \%$ (Yazaki y Aung, 1989) y de $88 \%$ (Inoue et al., 1998), por lo que de los extractos acuosos de las especies estudiadas en este trabajo, sólo el $P$. leiophylla alcanza valores similares al $P$. radiata (Tabla 1).

El contenido de taninos condensados es directamente proporcional al rendimiento en extracto total y al número de Stiasny. Los valores más bajos de taninos condensados fueron los de los extractos acuosos de $P$. cooperi y $P$. engelmannii, con tan solo el $1 \%$. En general, estos rendimientos fueron bajos en los extractos acuosos de todas las especies, el valor más alto se alcanzó en $P$. ayacahuite con $7,32 \%$. Sin embargo, en los extractos etanólicos de todas las especies los rendimientos fueron mucho mayores, con valores de $6,46 \%$ (P. arizonica) a $16,18 \%$ $(P$. leiophylla). La excepción fue $P$. engelmannii con 3,25\%. De acuerdo con el análisis estadístico se encontraron diferencias significativas $(p<0,05)$ entre los sistemas de extracción y entre las especies. En la Tabla 3 se presentan los valores medios del porcentaje de taninos condensados.

Se han determinado rendimientos en taninos condensados de 10,5\% para extractos acuosos de $P$. radiata (Inoue et al., 1998). Honorato y Hernández (1998), mencionan que las cortezas con un $8 \%$ de taninos pueden considerarse con potencial comercial para utilizarse como fuentes alternativas de taninos, por lo que se puede deducir que solamente los extractos etanólicos de las especies $P$. ayacahuite, $P$. durangensis, $P$. leiophylla y $P$. teocote pudieran ser viables para este fin.

Los rendimientos de fenoles totales en el extracto fueron menores en el acuoso con respecto al etanólico, aunque en algunas especies estos rendimientos fueron similares, como en $P$. leiophylla con $72,2 \%$ en el extracto acuoso y $78,5 \%$ en el extracto etanólico. Los rendimientos más bajos fueron los del extracto acuoso de $P$. cooperi con $37,5 \%$ y $P$. engelmannii con $32,2 \%$. Estadísticamente se encon-traron diferencias entre algunos de los extractos $y$ especies $(p<0,05)$, así como semejanzas entre otras, como en el caso 
de los extractos acuosos de $P$. durangensis $(72,20 \%)$ y $P$. leiophylla $(72,17 \%)$, así como $P$. ayacahuite $(64,30 \%)$ y $P$. chihuahuana (62,0 \%). En los extractos etanólicos no se encontraron diferencias estadísticas entre $P$. leiophylla $(78,50 \%)$ y
$P$. durangensis $(78,17 \%)$, ni entre $P$. teocote $(62,33 \%), P$. ayacahuite $(61,83$ $\%)$ y $P$. arizonica $(61,17 \%)$. En la Tabla 4 se resumen los valores de fenoles como ácido tánico y las diferencias.

Tabla 3. Tabla de medias del porcentaje de taninos condensados

\begin{tabular}{lclc}
\hline \multicolumn{2}{c}{ EXTRACTO ACUOSO } & \multicolumn{2}{c}{ EXTRACTO ETANÓLICO } \\
\hline Especie & Media ${ }^{*}$ & Especie & Media $^{*}$ \\
\hline$P$. ayacahuite & $7,32 \mathrm{a}$ & P. leiophylla & $16,18 \mathrm{a}$ \\
\hline$P$. durangensis & $6,46 \mathrm{~b}$ & P. ayacahuite & $15,50 \mathrm{~b}$ \\
\hline$P$. leiophylla & $5,87 \mathrm{c}$ & $P$. durangensis & $13,48 \mathrm{c}$ \\
\hline$P$. arizonica & $3,15 \mathrm{~d}$ & P. teocote & $9,12 \mathrm{~d}$ \\
\hline p. teocote & $3,03 \mathrm{~d}$ & P. chihuahuana & $7,20 \mathrm{e}$ \\
\hline$P$. chihuahuana & $2,46 \mathrm{e}$ & P. cooperi & $7,06 \mathrm{e}$ \\
\hline$P$. cooperi & $1,00 \mathrm{f}$ & P. arizonica & $6,47 \mathrm{f}$ \\
\hline$P$. engelmannii & $1,00 \mathrm{f}$ & P. engelmannii & $3,25 \mathrm{~g}$ \\
\hline
\end{tabular}

*Letras iguales significa que no existe diferencia estadística

Tabla 4. Tabla de medias del porcentaje de fenoles como ácido tánico

\begin{tabular}{lcll}
\hline \multicolumn{2}{c}{ EXTRACTO ACUOSO } & \multicolumn{1}{c}{ EXTRACTO ETANÓLICO } \\
\hline Especie & Media * & Especie & Media * \\
P. durangensis & $72,20 \mathrm{a}$ & P. chihuahuana & $81,67 \mathrm{a}$ \\
P. leiophylla & $72,17 \mathrm{a}$ & P. leiophylla & $78,50 \mathrm{~b}$ \\
P. ayacahuite & $64,30 \mathrm{~b}$ & P. durangensis & $78,17 \mathrm{~b}$ \\
P. chihuahuana & $62,00 \mathrm{~b}$ & P. teocote & $62,33 \mathrm{c}$ \\
P. teocote & $56,83 \mathrm{c}$ & P. ayacahuite & $61,83 \mathrm{~cd}$ \\
P. arizonica & $53,50 \mathrm{~d}$ & P. arizonica & $61,17 \mathrm{~cd}$ \\
P. cooperi & $37,50 \mathrm{e}$ & P. engelmannii & $59,50 \mathrm{~d}$ \\
P. engelmannii & $32,17 \mathrm{f}$ & P. cooperi & $49,17 \mathrm{e}$ \\
\hline
\end{tabular}

*Letras iguales significa que no existe diferencia estadística 


\section{CONCLUSIONES}

Existen diferencias estadísticas en la concentración de compuestos fenólicos entre las especies de pino estudiadas, así como entre los solventes de extracción utilizados. Los extractos etanólicos presentan un mayor rendimiento en extracto total, taninos condensados y fenoles totales, con respecto a los acuosos. Los extractos etanólicos de las cortezas de $P$. durangensis, $P$. ayacahuite y $P$. leiophylla, son los que presentan la mayor concentración de compuestos fenólicos, con porcentajes de taninos condensados de $13 \%$ a $16 \%$, lo que indica que poseen una considerable proporción de compuestos flavonoides, que les dan un buen potencial para su utilización en áreas biomédicas. Las especies que presentan la menor concentración de estos compuestos son $P$. engelmannii y $P$. cooperi.

\section{REFERENCIAS}

De Bruyne, T.; L. Pieters; H. Deelstra y A. Vlietinck. 1999. Condensed vegetable tannins: Biodiversity in structure and biological activities. Biochemical Systematics and Ecology 27:445-459.

Dey, P.M. y J.B. Harborne. 1989. Methods in plant biochemistry. Vol 1: Plant phenolics. Academic Press. 552 p.

Fengel, D. y G. Wegener. 1989. Wood, chemistry, ultrastructure, reactions. Walter de Gruyter Ed. Nueva York, p: 90-115.

García A., A. y S. González E. 1998. Pináceas de Durango. Instituto de Ecología-CIIDIR Unidad Durango. $179 \mathrm{p}$.

Garro G., J.; B. Riedl y H. Conner A. 1997. Analytical studies on tara tannins. Holzforschung 51(3):235-243.
González L., R.F.; G. Ochoa R.; N. Guzmán B. y E. Castañeda M. 1989. Utilización de taninos de corteza de pino en la preparación de adhesivos para vigas laminadas. UBAMARI 16:18-31.

Honorato S., J.A. y J. Hernández P. 1998. Determinación de componentes químicos de la madera de cinco especies de encino del estado de Puebla. Madera y Bosques 4(2):79-93.

Inoue, S.; M. Asaga; T. Ogi y Y. Yazaki. 1998. Extraction of polyflavonoids from radiata pine bark using hot compressed water al temperatures higher than $100{ }^{\circ} \mathrm{C}$. Holzforschung 52(2):139-145.

Kofujita, H.; K. Ettyu y M. Ota. 1999. Characterization of the major components in bark from five Japanese tree species for chemical utilization. Wood Science and Technology 33:223-228.

Laks E., P. y A.P. McKaig. 1988. Flavonoid biocides: wood preservatives based on condensed tannins. Holzforschung 42(5):299-306.

Peterson, J. y J. Dwyer. 2000. An informatics approach to flavonoid database development. Journal of food composition and analysis 13:441-454.

Pizzy, A. y K.L. Mittal. 1994. Handbook of adhesive technology. Marcel Dekker Inc. Nueva York, Basel, Hong Kong. $680 \mathrm{p}$.

Rosales C., M.; A. Galindo C. y R. González L. 2002. Taninos condensados en cortezas de dos especies de pino. Información Tecnológica (CIT), Chile 13(1):39-42.

Rosales C., M. 2002. Proyecto Evaluación de la actividad antioxidante de 
extractos de corteza de pino CIIDIR Durango. Financiado por la Coordinación General de Posgrado e Investigación del Instituto Politécnico Nacional, 20020443. En proceso.

Scalbert, A. 1992. Quantitative methods for the estimation of tannins in plant tissues. Plant polyphenols. Plenum Press, Nueva York. 450 p.

Van Sumere, C.F. 1989. Phenols and phenolic acids. Methods in plant biochemistry, Vol 1. Academic Press. p: 29-73.

Vázquez, G.; G. Antorrena y C. Parajó J. 1987. Studies on the utilization of Pinus pinaster bark. Wood Science and Technology 21:65-74.

Waterman, P.G. y S. Mole. 1994. Methods in ecology. Analysis of phenolic plant metabolites. Blackwell Scientific publications. 237p.

Yazaki, Y.; R. Gu; Y. Lin; W. Chen y K. Nguyen N. 1993. Analysis of black wattle (Acacia mearnsii) tanninsRelationships among the hidepowder, the Stiasny and the ultra violet (UV) methods. Holzforschung 47(1):57-61.

Yazaky, Y. y E.W. Hillis. 1980. Molecular size distribution of radiata pine bark extracts and its effect on propierties. Holzforschung 34:125-130.

Yazaki, Y. y T. Aung. 1989. Effect of $\mathrm{NaOH}$ on Stiasny values of extractives from Pinus radiata Bark. Holzforschung 43(4):281-282. $\diamond$

1 CIIDIR IPN Unidad Durango. Av Sigma s/n Fracc. 20 de Noviembre, Durango Durango. c.e.: mrosa0563@yahoo.com.

2 Instituto Tecnológico de Durango. Felipe Pescador 1830 Ote. Durango Durango. c.e.: ruben@scientist.com.

Manuscrito recibido el 3 de marzo de 2003.

Aceptado el 14 de julio de 2003.

Este documento se debe citar como:

Rosales C., M. y R.F. González L. 2003. Comparación del contenido de compuestos fenólicos en la corteza de ocho especies de pino. Madera y Bosques 9(2):41-49. 